\title{
Numerical modelling of caustics of solid state disk lasers
}

\author{
Marcin Kubiak ${ }^{*}, 1$ \\ ${ }^{1}$ Czestochowa University of Technology, Institute of Mechanics and Machine design Foundations, \\ Dabrowskiego 73, 42-200 Czestochowa, Poland
}

\begin{abstract}
This paper concerns mathematical modelling of caustics of solid state laser YAG type with the disk active medium. The heat source model is developed on the basis of interpolation algorithms using geostatistical kriging method. The real laser beam power distribution and caustics are taken into account in the model. Measurements of laser beam power distribution and diameter of the laser beam spot for different focusing are performed using UFF100 analyzer. Yb:YAG laser emitted by Trumpf laser head D70 is used in the experiment. Presented results include the comparison of experimentally determined laser power intensity distribution and caustic with results obtained by developed interpolation model.
\end{abstract}

Keywords: Yb:YAG laser, thermal load, heat source, Kriging algorithm, numerical modelling

\section{Introduction}

Changes in the focal length of focusing lens in the optical system of solid state laser changes not only the laser spot diameter but also the laser beam power intensity distribution. Beam divergence which is a measure for how fast the beam expands far from the beam waist is usually defined by the measurement of beam caustic - the beam radius at different positions. The measurement of laser beam power distribution is performed by using laser profilers [1-3].

In mathematical and numerical models of laser beam heat source power distribution only specific laser beam spot diameter is adopted, omitting the beam caustics. Generally, the distance from beam focusing position as a technological parameter influencing laser beam intensity distribution is usually neglected. Consequently, the laser beam intensity distribution models assumed in numerical analysis significantly differ from real Yb:YAG laser profile, obtained through experimental research [4, 5].

This paper presents interpolation algorithms allowing a precise description of Yb:YAG laser power intensity distribution and its caustic. Elaborated models take into account the real laser power distribution obtained in experimental research made using TruDisk 12002 laser. Kriging method [6-8] is used in this study in the form of point Kriging for the interpolation of laser beam heat source power distribution.

\footnotetext{
* Corresponding author: kubiak@imipkm.pcz.pl

Reviewers: Piotr Krawiec, Tomáś Lack
} 


\section{Experimental research}

The light is pumped to the laser beam head by optical fibers with core diameter $\mathrm{d}_{\text {LLK }}$. After the exit from the fiber, the beam is divergent by angle $\Theta_{L L K}$ to diameter $d_{0}$. The laser head is equipped with collimator lens having focal length $f_{c}=200 \mathrm{~mm}$ and focusing lens with focal length $f_{o g}=400 \mathrm{~mm}$. After focusing of laser light by focusing length by angle $\Theta_{f}$ the spot diameter (laser beam focus diameter) is equal to $d_{o g}$. The following equations can be used to determine average diameter of the laser beam focus, Rayleigh length $\left(Z_{R}\right)$ as well as the actual diameter $d(z)$ depending on beam focusing position $(z)$.

$$
\begin{gathered}
\mathrm{d}_{\mathrm{og}}=\frac{f}{f_{c}} d_{L L K} \\
\mathrm{Z}_{\mathrm{R}}=\frac{\pi\left(d_{o g} / 2\right)^{2}}{\lambda} \\
\mathrm{d}(\mathrm{z})=d_{o g} \sqrt{1+\left(\frac{z}{Z_{R}}\right)^{2}}
\end{gathered}
$$

where $\lambda$ is a wavelength of laser radiation.

The measurement of beam power distribution and caustics is performed using beam profiler Prometec UFF100 (Fig. 2). UFF100 is a beam profiler for high power lasers. It measures laser beam profile due to hollow needle. Laser beam is flowing through rotating needle and is transported to the detector. Detector signals are transformed into digital signals and processed later by computer software.

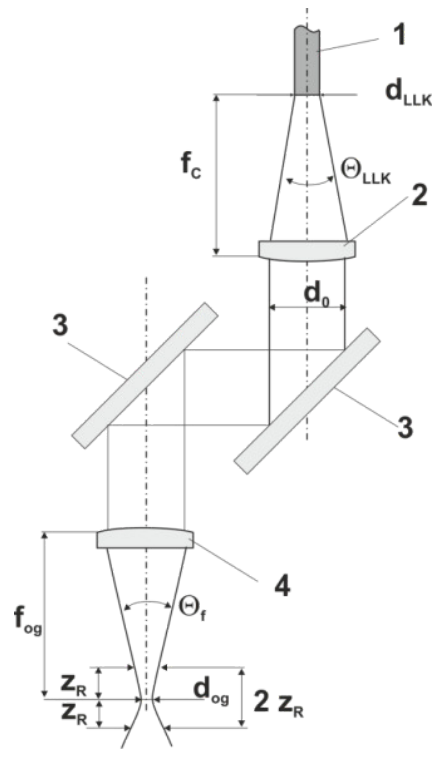

Fig. 1. Basic relations of laser beam transported through the fiber and focused by a laser head. 1 optical fiber, 2 collimator lens, 3 directional mirrors, 4 focusing lens

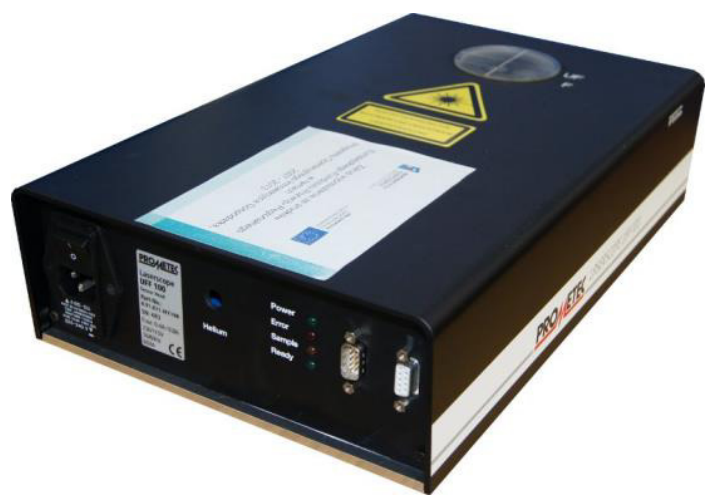

Fig. 2. Laser beam analyzer UFF100 
The power density distribution of the laser beam in a plane perpendicular to the axis of beam propagation (assumed to be $z$-axis) for different measurement planes is analyzed. The analysis is performed at the continuous power $900 \mathrm{~W}$ of the laser beam. The position of measurement plane changes with respect to the theoretical distance from the beam focusing position in the range of $\pm 10 \mathrm{~mm}$. For each testing planes, the radius of the beam in two perpendicular axes $\left(w_{x}\right.$ and $\left.w_{y}\right)$ is defined from which an average radius of the laser beam spot is calculated $(w)$ in accordance with standard PN-EN ISO 11146. Figure 3 presents experimentally obtained percentage distribution of $\mathrm{Yb}$ :YAG laser beam power for chosen beam focusing ( $\mathrm{z}=0$ and $\pm 5 \mathrm{~mm}$ ). Figure 4 illustrates the beam caustic $w_{x}, w_{y}$ and average radius $w$.

a)
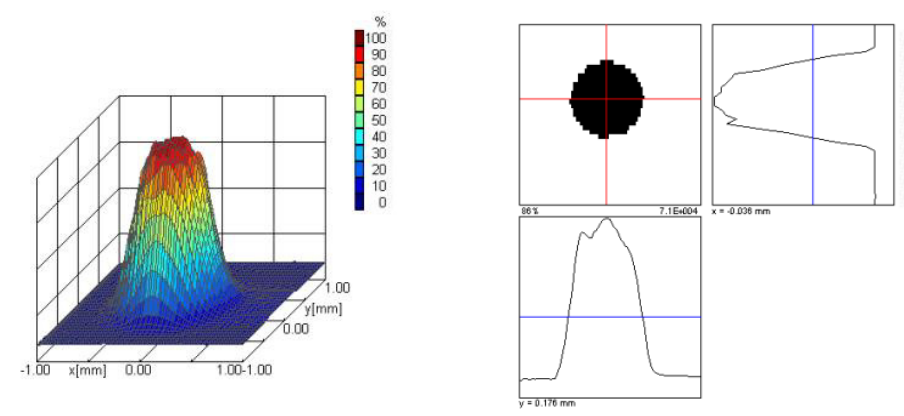

b)
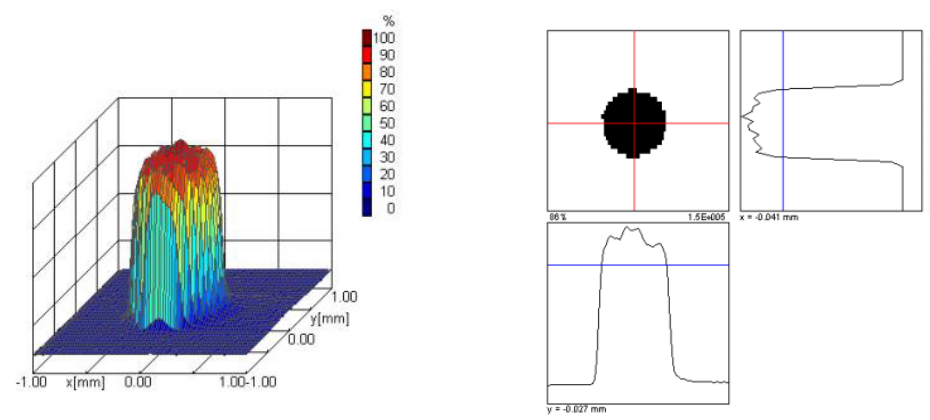

c)
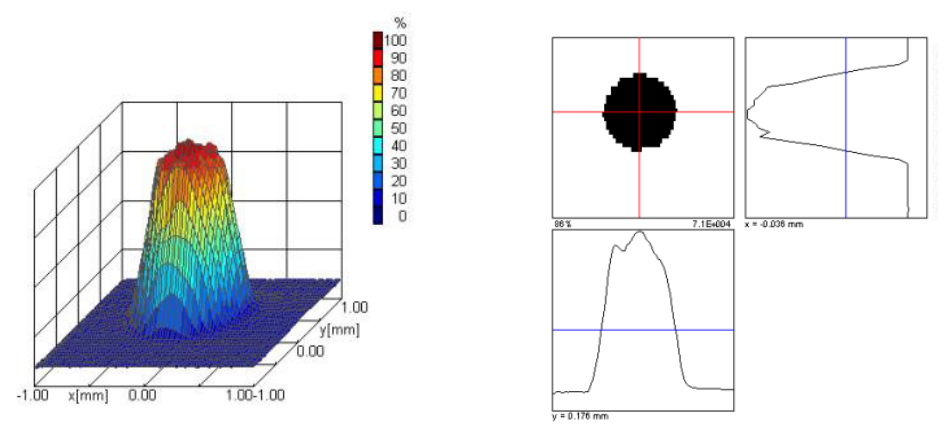

Fig. 3. Percentage laser beam power distribution at focusing a) $\mathrm{z}=5 \mathrm{~mm}, \mathrm{~b}) \mathrm{z}=0$ and c) $-5 \mathrm{~mm}$ 

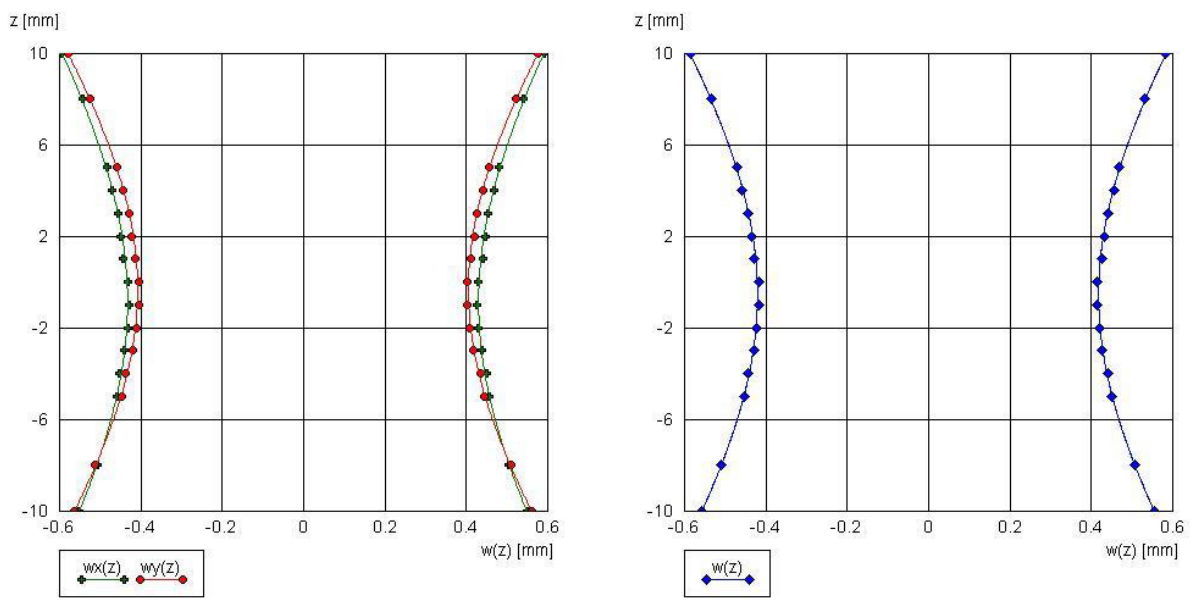

Fig. 4. Two dimensional picture of beam caustic: a) in perpendicular axes and b) average beam spot diameter

\section{Interpolated laser beam power distribution based on Kriging algorithm}

Kriging interpolation method of predicting the power distribution of the heat source at the point $(x, y)$ is a linear combination of observations in basic points (the actual power distribution). The estimate is a function of the weighted average:

$$
\tilde{f}(x, y)=\sum_{i=1}^{n} w_{i} f\left(x_{i}, y_{i}\right)
$$

where $w_{i}$ are weight coefficients assigned to particular observations, $f\left(x_{i}, y_{i}\right)$ is the real value of the function (variable) at the measured point, $n$ is the number of sampling points that are considered in estimating of the variable within the circle of radius $r_{k}$ from estimated point.

Coefficients $w_{i}$ are calculated on the basic of Kriging system of equations, as follows:

$$
\left[\begin{array}{cccccc}
0 & \gamma\left(h_{12}\right) & \gamma\left(h_{13}\right) & \ldots & \gamma\left(h_{1 n}\right) & 1 \\
\gamma\left(h_{21}\right) & 0 & \gamma\left(h_{23}\right) & \ldots & \gamma\left(h_{2 n}\right) & 1 \\
\gamma\left(h_{31}\right) & \gamma\left(h_{32}\right) & 0 & \ldots & \gamma\left(h_{3 n}\right) & 1 \\
\ldots & \ldots & \ldots & \ldots & \ldots & \ldots \\
\gamma\left(h_{n 1}\right) & \gamma\left(h_{n 2}\right) & \gamma\left(h_{n 3}\right) & \ldots & 0 & 1 \\
1 & 1 & 1 & \ldots & 1 & 0
\end{array}\right] \times\left[\begin{array}{c}
w_{1} \\
w_{2} \\
w_{3} \\
\ldots \\
w_{n} \\
\lambda
\end{array}\right]=\left[\begin{array}{c}
\gamma\left(d_{1}\right) \\
\gamma\left(d_{2}\right) \\
\gamma\left(d_{3}\right) \\
\ldots \\
\gamma\left(d_{n}\right) \\
1
\end{array}\right]
$$

where $\gamma\left(h_{i j}\right)$ are the values of theoretical semivariogram at the distance $h_{i j}$ between basis points in the observation set, $\gamma\left(d_{i}\right)$ are the values of theoretical semivariogram at the distance $d_{i}$ between observed point and $i$-th basic point, $\lambda$ is Lagrange multiplier.

Theoretical semivariogram is generally unknown, therefore values of function $\gamma\left(h_{i j}\right)$ and $\gamma\left(d_{i}\right)$ are approximated by analytical functions. One of the main function used in the approximation is a linear function $\gamma(\mathrm{h})=C_{0}+S h$, in which the semivariogram tends to sill 
$S$ at $h \rightarrow \infty$, while $C_{0}$ is a function discontinuity (nugget effect). Coefficients $S$ and $C_{0}$ are estimated using empirical semivariogram and sample variance Var, described by the following relations:

$$
\begin{aligned}
& \widehat{\gamma}\left(\mathrm{h}_{\mathrm{k}}\right)=\frac{1}{2 \mathrm{n}} \sum_{i, j=1}^{n}\left(f\left(x_{i}, y_{i}\right)-f\left(x_{j}, y_{j}\right)\right)^{2} \\
& \operatorname{Var}=\frac{1}{n-1} \sum_{i=1}^{\mathrm{n}}\left(f\left(x_{i}, y_{i}\right)-\bar{f}\right)^{2}, \quad \bar{f}=\frac{1}{n} \sum_{i=1}^{\mathrm{n}} f\left(x_{i}, y_{i}\right)
\end{aligned}
$$

where $\mathrm{n}=\mathrm{n}\left(\mathrm{r}_{\mathrm{k}}\right)$ is the number of basic points in a measurement set, $\bar{f}$ is the average value of the function in a measurement set.

Finally, after solving the system of equations the heat source power distribution for interpolation grid is calculated as follows:

$$
q(x, y, z)=\eta Q_{S} \tilde{f}(x, y)
$$

\section{Results and discussion}

Figures 5-6 show the power distribution of the heat source obtained by Kriging method for interpolation grid step $\Delta h=0.02 \mathrm{~mm}$ taking into account experimental data for $z=0$ and at the distance from beam focusing position $\mathrm{z}=10 \mathrm{~mm}$ respectively. In all figures the comparison of modelled percentage power intensity distribution with experimentally obtained distribution is illustrated in central axes of the heat source $(x=0$ and $y=0)$.

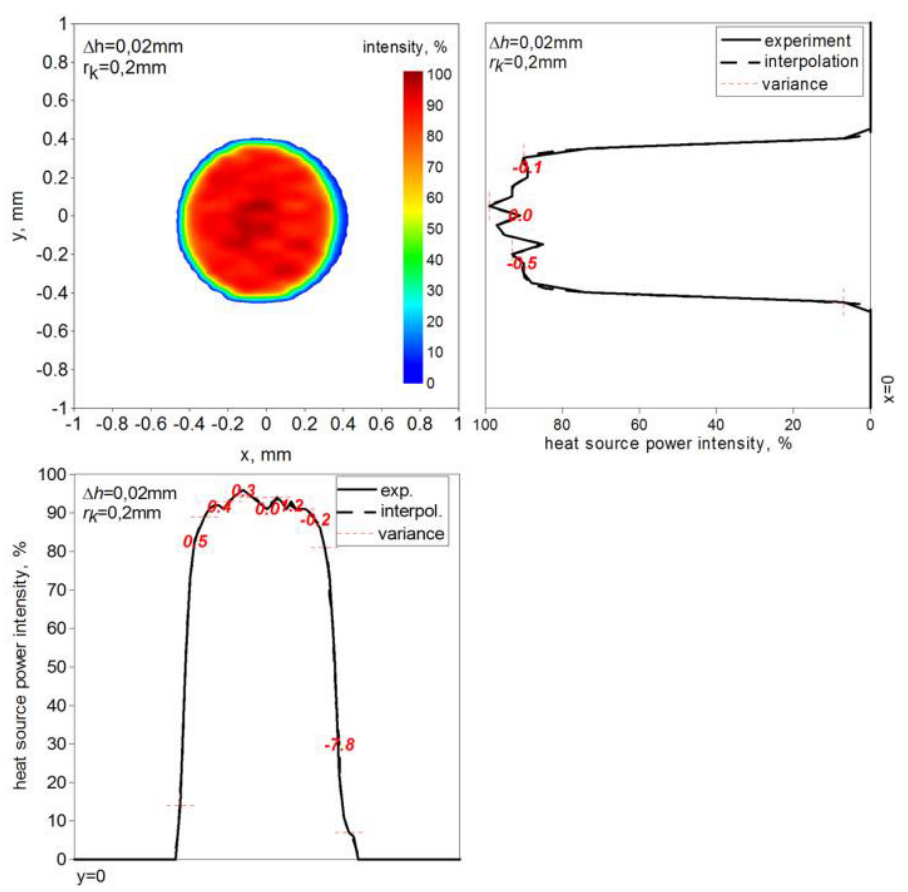

Fig. 5. Percentage distribution of laser power described by interpolation model for $\Delta \mathrm{h}=0.02 \mathrm{~mm}$. Distance from beam focusing $z=0$ 


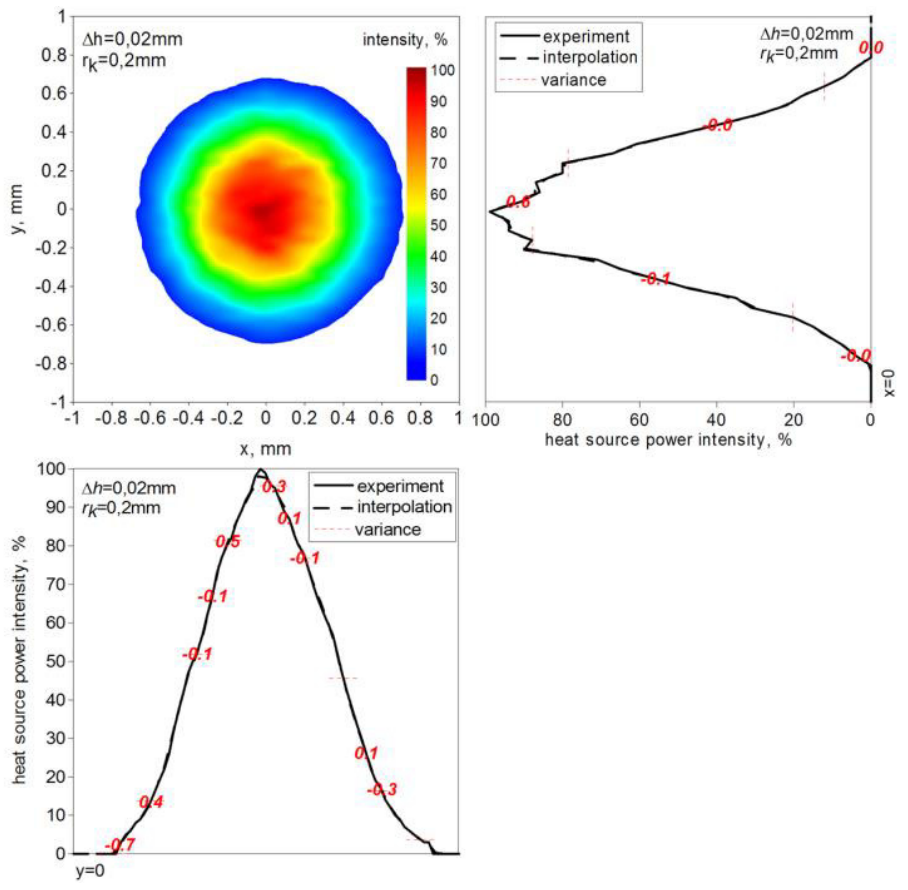

Fig. 6. Percentage distribution of laser power described by interpolation model for $\Delta \mathrm{h}=0.02 \mathrm{~mm}$.

Distance from beam focusing $z=10 \mathrm{~mm}$

Equations 1-3 allowed the prediction of beam caustic for $f_{\text {col }}=200 \mathrm{~mm}, f_{\text {og }}=400 \mathrm{~mm}$ and different diameters of optical fibers. Calculated beam caustic is presented in Fig. 7.

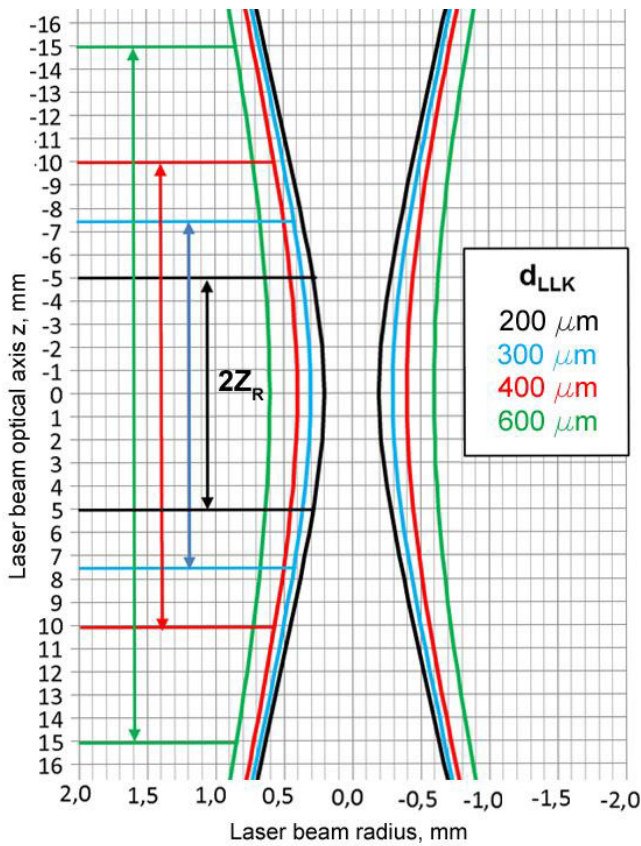

Fig. 7. Predicted beam caustic for different fibres diameters $d_{L L K}$ 


\section{Conclusions}

The increase of the distance from the beam focusing position changes the nature of intensity distribution, from top-hat to Gaussian like distribution. Therefore, classical Gauss distribution don't fit such laser beam power intensity distribution. The modelling of Yb:YAG laser power distribution by interpolation algorithms allows for a precise reproduction of the real thermal load, depending on the laser profile obtained for specified industrial laser.

Interpolation model can include technological parameter like the distance from the beam focusing position " $z$ " for which suitable heat source distribution is selected. This model takes also into account local extremes existing in the distribution.

Developed model allows considering not only the influence of the distance from beam focusing on the laser spot diameter but also on the laser beam power intensity distribution at different distances from beam focusing position.

\section{References}

1. D. Richardson, J. Nilsson, W. Clarkson, High power fiber lasers: current status and future perspectives. Journal of the Optical Society of America B.27, 63-92 (2010)

2. T. Y. Fan, Heat generation in Nd:YAG and Yb:YAG. IEEE Journal of Quantum Electronics 29, 1457-1459 (1993)

3. R. Brockmann, D. Havrilla, Third generation of disk lasers. A new benchmark for industrial solid state lasers. Laser Technik Journal 6 (3), 26-31 (2009)

4. X. Song, B. Li, Z. Guo, S. Wang, D. Cai, J. Wen, Influences of pump beam distribution on thermal lensing spherical aberration in an LD end-pumped Nd:YAG laser. Optics Communications 282, 4779-4783 (2009)

5. C. Pfistner, R. Weber, H. P. Weber, S. Merazzi, R. Gruber, Thermal beam distortions in end-pumped Nd:YAG, Nd: GSGG, and Nd: YLF Rods. IEEE Journal of Quantum Electronics 30, 1605-1614 (1994)

6. M. A. Oliver, R. Webster, Kriging: a method of interpolation for geographical information system. Int J Geo Inf Sys. 4 (3), 313-332 (1990)

7. S. Sakata, F. Ashida, M. Zako, Structural optimization using Kriging approximation. Computational Methods in Applied Mechanical Engineering 192 (7-8), 923-939 (2003)

8. M. Kubiak, W. Piekarska, Z. Saternus, T. Domański, Numerical prediction of fusion zone and heat affected zone in hybrid Yb: YAG laser+ GMAW welding process with experimental verification. Procedia Engineering 136, 88-94 (2016) 